\title{
Whole-genome sequencing of clarithromycin resistant Helicobacter pylori characterizes unidentified variants of multidrug resistant efflux pump genes
}

\author{
Akira Iwamoto ${ }^{1}$, Toshihito Tanahashi ${ }^{1} 2^{*}$, Rina Okada ${ }^{1}$, Yukio Yoshida ${ }^{3}$, Kaoru Kikuchi ${ }^{3}$, Yoshihide Keida ${ }^{3}$,
} Yoshiki Murakami ${ }^{4}$, Lin Yang ${ }^{1}$, Koji Yamamoto ${ }^{1}$, Shin Nishiumi ${ }^{1}$, Masaru Yoshida ${ }^{1}$ and Takeshi Azuma ${ }^{1}$

\begin{abstract}
Background: Clarithromycin (CLR) is the key drug in eradication therapy of Helicobacter pylori (H. pylori) infection, and widespread use of CLR has led to an increase in primary CLR-resistant H. pylori. The known mechanism of CLR resistance has been established in A2146G and A2147G mutations in the 23S rRNA gene, but evidence of the involvement of other genetic mechanisms is lacking. Using the MiSeq platform, whole-genome sequencing of the 19 clinical strains and the reference strain ATCC26695 was performed to identify single nucleotide variants (SNVs) of multi-drug resistant efflux pump genes in the CLR-resistant phenotype.
\end{abstract}

Results: Based on sequencing data of ATCC26695, over one million sequencing reads with over 50-fold coverage were sufficient to detect SNVs, but not indels in the bacterial genome. Sequencing reads of the clinical isolates ranged from 1.82 to 10.8 million, and average coverage ranged from 90.9- to 686.3-fold, which were acceptable criteria for detecting SNVs. Utilizing the conventional approach of allele-specific PCR, point mutations in the $23 \mathrm{~S}$ rRNA gene were detected in 12 clinical resistant isolates, but not in 7 clinical susceptible isolates. All sequencing reads of CLR-resistant strains had a $\mathrm{G}$ mutation in an identical position of the $23 \mathrm{~S}$ rRNA gene. In addition, genetic variants of four gene clusters (hp0605-hp0607, hp0971-hp0969, hp1327-hp1329, and hp1489-hp1487) of TolC homologues, which have been implicated in multi-drug resistance, were examined. Specific SNVs were dominantly found in resistant strains.

Conclusions: Gene clusters of TolC homologues are involved in CLR susceptibility profiles in individual H. pylori strains. Whole-genome sequencing has yielded novel understanding of genotype-phenotype relationships.

Keywords: Whole-genome sequencing, Helicobacter pylori, Clarithromycin, Multidrug efflux, TolC homolog

\section{Background}

Helicobacter pylori (H. pylori) infection is recognized in approximately $50 \%$ of the adult population and associated with a wide range of upper gastrointestinal diseases [1,2]. Present treatments for $H$. pylori infection consist of a proton pump inhibitor based triple therapy in combination

\footnotetext{
* Correspondence: tana@kobepharma-u.ac.jp

'Department of Internal Medicine, Division of Gastroenterology, Kobe University Graduate School of Medicine, 7-5-1 Kusunoki-cho, Chuo-ku, Kobe 650-0017, Japan

${ }^{2}$ Department of Medical Pharmaceutics, Kobe Pharmaceutical University,

4-19-1 Motoyama-kita, Higashinada-ku, Kobe 658-8558, Japan

Full list of author information is available at the end of the article
}

with amoxicillin and clarithromycin (CLR) [2]. Triple therapy has remained the first-choice regimen for the past decade and has been recommended by most consensus meetings, and by both European and American scientific societies [3-6].

In many cases, CLR is the key component of eradication therapy, but antibiotic resistance is the primary failure of triple therapy in patients with $H$. pylori infection. Prevalence of CLR-resistant $H$. pylori has become increasingly common in many countries $[7,8]$. Consequently, it has been suggested that the efficacy of standard triple therapy has been gradually reduced to unacceptable levels [9]. It is 
accepted that triple therapy should not be used to manage $H$. pylori infection when the prevalence of CLR resistance is higher than $15-20 \%$ [3]; in such cases, other therapeutic alternatives should be considered.

CLR binds to the peptidyl transferase region of the bacterial 23S rRNA and inhibits protein synthesis [10]. Acquired resistance of CLR in $H$. pylori has been associated with point mutations in the peptidyl transferase region of domain $\mathrm{V}$ of the $23 \mathrm{~S}$ rRNA. Two copies of the 23S rRNA gene are present in $H$. pylori, and the most common mutation is an A-to-G transition at position 2146 and 2147 (previously known as A2142G and A2143G) [11]. Recent reports have also indicated that other $23 \mathrm{~S}$ rRNA gene mutations might be associated with CLR resistance [12,13]. In addition, CLR resistance seems to be related with other factors, such as rRNA methylase production [14], the actions of macrolide-inactivating enzymes, and active efflux pumps, which have been described in several bacterial species [15]. Indeed, an active efflux pump has been shown to contribute to CLR resistance in $H$. pylori [16]. As a result of the worldwide increased rate of CLR resistance, some studies have indicated that other bacterial genetic factors might contribute to this increased resistance $[17,18]$. However, there is no genetic evidence for other bacterial factors in $H$. pylori.

To characterize the bacterial pathogen samples, recent advances in whole-genome sequencing, such as the development of DNA-sequencing technologies, were applied to this study [19]. Compared to conventional methods (e.g., capillary sequencing and non-sequencebased molecular methods), next-generation sequencing provides deep views on bacterial genome information without biases in downstream analysis [20]. These techniques are the ideal tool to comprehensively track extensive genomic knowledge of antimicrobial resistance and to facilitate specific and rational treatment of infected patients [21]. However, despite the broad advantages, next-generation sequencing requires the extensive bioinformatics tasks for data analysis from assembly to gene annotation, and this remains a significant problem for the routine application to clinical microbiology [22].

In this study, we first applied the established method of allele-specific PCR to detect 23S rRNA gene mutations in clinical $H$. pylori strains and identified CLR-resistant isolates. To elucidate the unidentified genetic factors in the reduced susceptibility to CLR in $H$. pylori, whole-genome sequencing was applied to 20 selected $H$. pylori strains, including the major laboratory strain ATCC26695, 12 resistant and 7 susceptible strains. Of note, we focused on variants of multidrug resistance (MDR) efflux pumps that contribute to both intrinsic and acquired antibiotic resistance and assessed their association with novel mechanisms of CLR resistance.

\section{Results}

AS-PCR for A2146G and A2147G mutations in 23S rRNA

For 19 clinical isolates, AS-PCR clearly differentiated the $23 \mathrm{~S}$ rRNA mutants from the $H$. pylori (Figure 1). Point mutations in the 23S rRNA gene at position 2146 or 2147 were detected in 12 strains and defined as CLR-resistant isolates in this study. Twelve mutant strains were isolated from patients who failed eradication therapy, and 7 wild-type strains were isolated from patients with successful eradication therapy. Thus, AS-PCR results were in agreement with the outcome of eradication therapy based on clinical information (data not shown).

\section{ATCC26695 total reads and average coverage}

Prior to analysis of clinical isolate sequencing data, we generated six independent sequencing data sets from ATCC26695 to determine the adequate total reads and average coverage that should be targeted for our study (Table 1). Illumina MiSeq generated 150-bp paired-end reads with 110,650 to $2,148,576$ reads from the six samples. After trimming, the number of total reads was nearly unchanged before trimming. When all data were mapped to the theoretical nucleotide length of ATCC26695 $(1,667,867 \mathrm{bp})$ using Genomics Workbench 6.0.1, the total consensus coverage (\%) ranged from 87.38 to $99.99 \%$, and average coverage was from 4.2 - to 81.7 -fold. In the sequencing data from sample 5 and 6 , each with over one million reads, we found nearly equal total consensus coverage (99.98 and 99.99\%, respectively) and average coverage (71.8- and 81.7-fold, respectively).

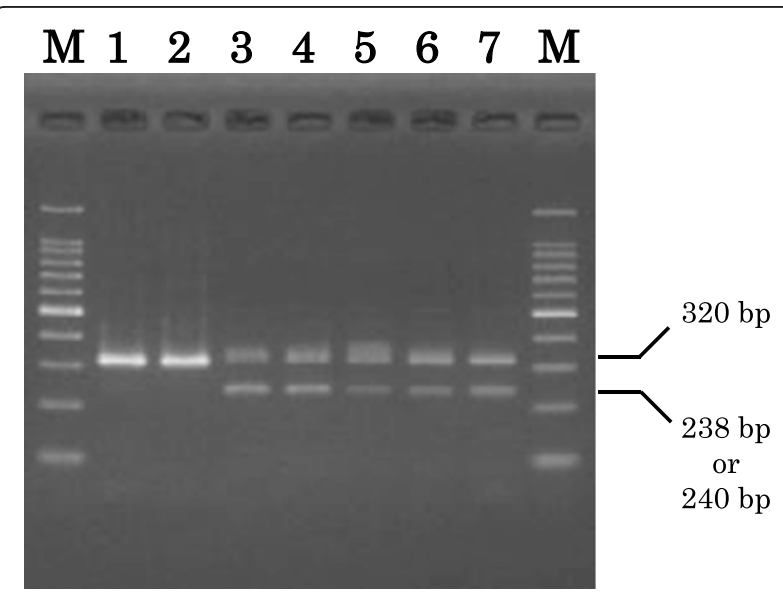

Figure 1 Representative electrophoresis patterns of allele-specific PCR products. PCR products were amplified with mixed primers for determination of A2146G and A2147G mutations in 23S rRNA of H. pylori. PCR products of $320 \mathrm{bp}$ are commonly observed for the $\mathrm{H}$. pylori $23 \mathrm{~S}$ rRNA gene. PCR products of 238 or 240 bp indicate the presence of a point mutation in the 235 rRNA gene. PCR products were obtained from ATCC26695 (lane 1), $J 99$ (lane 2), and five representative CLR-resistant strains (lanes 3-7; S1, S13, S17, S26, and 174). The 100 bp marker ladder is indicated by $\mathrm{M}$. 
Table 1 Six ATCC26695 sequence data sets

\begin{tabular}{llllllll}
\hline Sample ID & $\begin{array}{l}\text { Total reads } \\
\text { (before trimming) }\end{array}$ & $\begin{array}{l}\text { Total reads } \\
\text { (after trimming) }\end{array}$ & $\begin{array}{l}\text { Total consensus } \\
\text { length (bp) }\end{array}$ & $\begin{array}{l}\text { Total consensus } \\
\text { coverage (\%) }\end{array}$ & $\begin{array}{l}\text { Average } \\
\text { coverage (fold) }\end{array}$ & $\begin{array}{l}\text { Assemble } \\
\text { gaps (bp) }\end{array}$ & $\begin{array}{l}\text { NCBI accession } \\
\text { number }\end{array}$ \\
\hline 1 & 110,650 & 109,952 & $1,457,400$ & 87.38 & 4.2 & 210,467 & $\mathrm{NA}$ \\
2 & 130,226 & 130,216 & $1,649,483$ & 98.89 & 5.9 & 18,384 & $\mathrm{NA}$ \\
3 & 488,824 & 488,724 & $1,667,405$ & 99.97 & 29.5 & 462 & $\mathrm{NA}$ \\
4 & 664,278 & 642,532 & $1,667,519$ & 99.97 & 36.3 & 348 & $\mathrm{NA}$ \\
5 & $1,211,740$ & $1,195,756$ & $1,667,544$ & 99.98 & 71.8 & 323 & $\mathrm{NA}$ \\
6 & $2,148,576$ & $2,107,718$ & $1,667,758$ & 99.99 & 81.7 & 211 & DRS013117 \\
\hline
\end{tabular}

Total reads after trimming are mapped to the reference nucleotides of 1,667,867 bp (NC_000915) and calculated with Genomic Workbench 6.0.1. NA; not available.

\section{Quality control of ATCC26695 sequencing data}

Adapter sequences were automatically trimmed from the reads with MiSeq reporter analysis. As shown in Table 1, the sequencing data from sample 6 of ATCC26695 was subjected to the quality control analysis.

As a measure of quality control, we determined the length distribution of sequencing reads, ambiguous basecontent, and quality distribution at each base position (Additional file 1: Figure S1). This analysis led to the identification and subsequent removal of several errors in the raw sequencing data. After trimming, histograms of sequencing lengths distribution shifted to a shorter sequence length, and there was no ambiguous base-content. The mean values of Phred scores were $>30$ at all base positions up to $150 \mathrm{bp}$. Thus, quality control assessment showed that the entire length from the 150-bp paired-end reads could be analyzed. The FASTQ file, containing the trimmed sequences, was used for further analysis.

\section{Coding sequence from ATCC26695 sequencing data}

ATCC26695 sample 6 was chosen for further analysis based on its total number of reads, read quality, and coverage. Sequencing reads were aligned to the NC_000915 genome using Genomic Workbench 6.0.1. Analytical procedure was shown in Additional file 2: Figure S2 as a flow chart.

Despite over two million sequencing reads of ATCC26695, a total of 211 bp was identified as an assembly gap to the NC_000915 reference sequence (Table 2). The detailed assemble gaps were as follows; a total of 51 bp in 48 short insertions, 27 bp in 25 short deletions, and $133 \mathrm{bp}$ in 4 long deletions. The quality and coverage of the sequence data was effective to determine the differences between isolates of a single laboratory strain. In our sequencing data, seven nucleotides per genome $(1.66 \mathrm{Mb})$ were different from the reference nucleotides with over 50\% frequency (Table 3).

Taken together, these sequencing data indicated that over one million sequencing reads had $>50$-fold average coverage of the assembled contigs when reads were mapped to the reference $H$. pylori genome. Since it was difficult to identify true single-base and small indels during our mapping process, we did not try to identify any size of indels in further analysis of the clinical isolates.

\section{Whole-genome sequencing of 19 clinical $H$. pylori strains}

All H. pylori strains were sequenced with illumina MiSeq. After triminng, total reads of 7 wild-type (susceptible) strains ranged from 1.96 million to 5.32 million (Table 4A). Similarly, total reads of 12 resistant strains ranged from 1.82 million to 10.8 million (Table 4B). After trimming above-mentioned, sequencing data were mapped to the ATCC26695 reference genome. Average coverage ranged from 90.9 to 283.6-fold in susceptibile strains and from 125.5 to 686.3 -fold in resistant strains. These data indicated that the derived high sequencing depth of coverage was sufficient to detect high quality SNVs in the $H$. pylori genome.

\section{Detection of A2146G and A2147G with sequencing reads}

Prior to analyzing the target genes in the derived sequence data, we examined the position of point mutations in $23 \mathrm{~S}$ rRNA to identify any false positive variants. Two identical copies of 23S rRNA (HPr01 and HPr06) are found in the H. pylori genome [11].

Sequence data of 12 resistant strains mapped to the ATCC26695 reference genome. The results of two representative resistant strains, S17 and S26, are shown in Figure 2. In the S17 strain at position 2146 in the $23 \mathrm{~S}$ rRNA gene, all sequence reads (479-fold) had the variant G. Similarly, in S26 at position 2147, all reads (485-fold) had the variant G. In the other copy of the 23S rRNA, the same changes were found in each strain. Sequencing reads of the remaining 10 strains also contained the variant G. The AS-PCR is a targeted approach for identifying variants at one basepair position (i.e., 2146 or 2147), but whole-genome sequencing can simultaneously detect multiple base-pair variants with high confidence. In contrast, there was no variant in the same positions of 23S rRNA genes of 7 CLR-susceptible strains. Representative result of F79 strain was shown in Additional file 3: Figure S3. 
Table 2 Assemble gaps of ATCC26695 sequence data from sample 6

\begin{tabular}{|c|c|c|c|c|c|c|c|c|}
\hline \multirow[t]{2}{*}{ Type } & \multirow[t]{2}{*}{ Length (bp) } & Sequence & \multicolumn{2}{|c|}{$\begin{array}{l}\text { Nucleotide position in } \\
\text { reference sequence }\end{array}$} & \multirow[t]{2}{*}{ Locus tag } & \multirow[t]{2}{*}{$\begin{array}{l}\text { Locus_tag } \\
\left(5^{\prime} ? 3^{\prime}\right)\end{array}$} & \multirow[t]{2}{*}{ Annotation } & \multirow{2}{*}{$\begin{array}{l}\text { Coverage } \\
\text { Insertion: consensus side/ } \\
\text { Deletion: reference side }\end{array}$} \\
\hline & & Insertion Deletion & Start & End & & & & \\
\hline \multicolumn{9}{|c|}{ Short insertion } \\
\hline 1 & 1 & $A$ & 39762 & 39763 & HP0041 & $\rightarrow$ & hypothetical protein & 63 \\
\hline 2 & 1 & G & 159581 & 159582 & HP0147 & $\rightarrow$ & $\begin{array}{l}\text { cytochrome c oxidase, diheme subunit, } \\
\text { membrane-bound (fixP) }\end{array}$ & 89 \\
\hline 3 & 1 & $C$ & 173256 & 173257 & HP0165 & $\leftarrow$ & hypothetical protein & 94 \\
\hline 4 & 1 & G & 208167 & 208168 & HP0203 & $\rightarrow$ & hypothetical protein & 85 \\
\hline 5 & 1 & C & 263286 & 263287 & HP0253 & $\rightarrow$ & hypothetical protein & 105 \\
\hline 6 & 1 & C & 331675 & 331676 & & & & 2 \\
\hline 7 & 1 & T & 331678 & 331679 & & & & 2 \\
\hline 8 & 1 & G & 331680 & 331681 & & & & 2 \\
\hline 9 & 1 & $\mathrm{~T}$ & 331682 & 331683 & & & & 2 \\
\hline 10 & 1 & G & 474218 & 474219 & & & & 88 \\
\hline 11 & 1 & C & 475466 & 475467 & HP0456 & $\rightarrow$ & hypothetical protein & 53 \\
\hline 12 & 1 & C & 475645 & 475646 & & & & 46 \\
\hline 13 & 1 & T & 524751 & 524752 & HP0498 & $\rightarrow$ & sodium- and chloride-dependent transporter & 54 \\
\hline 14 & 1 & G & 579891 & 579892 & & & & 76 \\
\hline 15 & 1 & A & 626216 & 626217 & & & & 57 \\
\hline 16 & 1 & A & 674290 & 674291 & HP0628 & $\rightarrow$ & hypothetical protein & 63 \\
\hline 17 & 1 & C & 683744 & 683745 & HP0636 & $\leftarrow$ & hypothetical protein & 54 \\
\hline 18 & 1 & G & 739962 & 739963 & HP0688 & $\rightarrow$ & hypothetical protein & 45 \\
\hline 19 & 1 & T & 745341 & 745342 & HP0694 & $\rightarrow$ & hypothetical protein & 84 \\
\hline 20 & 1 & T & 745352 & 745353 & & & & 86 \\
\hline 21 & 1 & A & 745389 & 745390 & & & & 95 \\
\hline 22 & 1 & T & 745429 & 745430 & & & & 97 \\
\hline 23 & 2 & AG & 773423 & 773424 & HP0719 & $\rightarrow$ & hypothetical protein & 55 \\
\hline 24 & 1 & C & 787709 & 787710 & HP0732 & $\leftarrow$ & hypothetical protein & 62 \\
\hline 25 & 1 & G & 808800 & 808801 & fliD & $\rightarrow$ & flagellar capping protein & 57 \\
\hline 26 & 1 & C & 838354 & 838355 & HP0783 & $\leftarrow$ & hypothetical protein & 74 \\
\hline 27 & 1 & A & 843491 & 843492 & & & & 69 \\
\hline 28 & 1 & G & 861042 & 861043 & HP0807 & $\leftarrow$ & iron(III) dicitrate transport protein (fecA) & 86 \\
\hline
\end{tabular}


Table 2 Assemble gaps of ATCC26695 sequence data from sample 6 (Continued)

\begin{tabular}{|c|c|c|c|c|c|c|c|c|}
\hline 29 & 1 & G & 888773 & 888774 & HP0836 & $\rightarrow$ & hypothetical protein & 98 \\
\hline 30 & 2 & $A G$ & 915893 & 915894 & HP0863 & $\leftarrow$ & hypothetical protein & 112 \\
\hline 31 & 1 & $A$ & 915897 & 915898 & HP0863 & $\leftarrow$ & hypothetical protein & 112 \\
\hline 32 & 1 & C & 993909 & 993910 & HP0931 & $\rightarrow$ & hypothetical protein & 109 \\
\hline 33 & 1 & G & 1026924 & 1026925 & & & & 63 \\
\hline 34 & 1 & T & 1057531 & 1057532 & & & & 69 \\
\hline 35 & 1 & $\mathrm{~T}$ & 1057599 & 1057600 & & & & 59 \\
\hline 36 & 1 & $\mathrm{~T}$ & 1077873 & 1077874 & HP1014 & $\rightarrow$ & 7-alpha-hydroxysteroid dehydrogenase & 83 \\
\hline 37 & 1 & G & 1207116 & 1207117 & HP1144 & $\rightarrow$ & hypothetical protein & 74 \\
\hline 38 & 1 & C & 1207608 & 1207609 & HPrO4 & $\leftarrow$ & $16 S$ ribosomal RNA & 101 \\
\hline 39 & 1 & G & 1208482 & 1208483 & HPr04 & $\leftarrow$ & $16 \mathrm{~S}$ ribosomal RNA & 1 \\
\hline 40 & 1 & C & 1208483 & 1208484 & HPrO4 & $\leftarrow$ & $16 \mathrm{~S}$ ribosomal RNA & 2 \\
\hline 41 & 1 & $\mathrm{~T}$ & 1256328 & 1256329 & HP1186 & $\rightarrow$ & carbonic anhydrase & 72 \\
\hline 42 & 1 & C & 1259151 & 1259152 & & & & 113 \\
\hline 43 & 1 & A & 1264196 & 1264197 & HP1193 & $\rightarrow$ & aldo/keto reductase & 92 \\
\hline 44 & 1 & G & 1291618 & 1291619 & HP1216 & $\leftarrow$ & hypothetical protein & 74 \\
\hline 45 & 2 & GG & 1475694 & 1475695 & HPr06 & $\leftarrow$ & $23 \mathrm{~S}$ ribosomal RNA & 1 \\
\hline 46 & 1 & C & 1511030 & 1511031 & & & & 4 \\
\hline 47 & 1 & C & 1511162 & 1511163 & HPrO4 & $\leftarrow$ & $16 S$ ribosomal RNA & 83 \\
\hline 48 & 1 & C & 1511398 & 1511399 & HPrO4 & $\leftarrow$ & $16 \mathrm{~S}$ ribosomal RNA & 11 \\
\hline Total & 51 & & & & & & & \\
\hline \multicolumn{9}{|c|}{ Short deletion } \\
\hline 1 & 1 & A & 25622 & & & & & 58 \\
\hline 2 & 1 & C & 38695 & & HP0039m & $\rightarrow$ & conjugal plasmid transfer system protein & 62 \\
\hline 3 & 2 & YC & 129158 & 129159 & & & & 1 \\
\hline 4 & 1 & C & 342188 & & HP0326 & $\rightarrow$ & CMP-N-acetylneuraminic acid synthetase & 86 \\
\hline 5 & 1 & $\mathrm{~T}$ & 426389 & & HP0413 & $\leftarrow$ & transposase-like protein, PS3IS & 18 \\
\hline 6 & 1 & A & 463967 & & HP0445 & $\leftarrow$ & hypothetical protein & 75 \\
\hline 7 & 1 & A & 593944 & & & & & 81 \\
\hline 8 & 1 & G & 650897 & & HP0609 & $\rightarrow$ & hypothetical protein & 73 \\
\hline 9 & 1 & A & 674221 & & HP0627 & $\rightarrow$ & hypothetical protein & 47 \\
\hline 10 & 1 & $\mathrm{~T}$ & 728119 & & HP0678 & $\leftarrow$ & hypothetical protein & 75 \\
\hline
\end{tabular}


Table 2 Assemble gaps of ATCC26695 sequence data from sample 6 (Continued)

\begin{tabular}{|c|c|c|c|c|c|c|c|c|}
\hline 11 & 1 & G & 745422 & & & & & 98 \\
\hline 12 & 1 & C & 815405 & & HP0760 & $\leftarrow$ & phosphodiesterase & 41 \\
\hline 13 & 1 & C & 815420 & & HP0760 & $\leftarrow$ & phosphodiesterase & 48 \\
\hline 14 & 1 & $\mathrm{R}$ & 1027030 & & & & & 73 \\
\hline 15 & 1 & G & 1049706 & & & & & 58 \\
\hline 16 & 1 & G & 1071033 & & HP1009 & $\leftarrow$ & site-specific recombinase & 77 \\
\hline 17 & 1 & T & 1077870 & & HP1014 & $\rightarrow$ & 7-alpha-hydroxysteroid dehydrogenase & 87 \\
\hline 18 & 1 & G & 1081560 & & HP1018 & $\rightarrow$ & hypothetical protein & 97 \\
\hline 19 & 1 & G & 1192591 & & HP1127 & $\leftarrow$ & hypothetical protein & 61 \\
\hline 20 & 1 & $\mathrm{~T}$ & 1256382 & & & & & 81 \\
\hline 21 & 1 & C & 1258321 & & HP1188 & $\leftarrow$ & hypothetical protein & 71 \\
\hline 22 & 1 & T & 1264200 & & HP1193 & $\rightarrow$ & aldo/keto reductase & 91 \\
\hline 23 & 2 & NA & 1480608 & 1480609 & HP1410 & $\rightarrow$ & hypothetical protein & 1 \\
\hline 24 & 1 & A & 1480611 & & HP1410 & $\rightarrow$ & hypothetical protein & 1 \\
\hline 25 & 1 & A & 1480614 & & HP1410 & $\rightarrow$ & hypothetical protein & 1 \\
\hline Total & 27 & & & & & & & \\
\hline \multicolumn{9}{|c|}{ Long deletion } \\
\hline 1 & 42 & $\begin{array}{l}\text { TGATTATATTTGTAATGGTGCTC } \\
\text { RCTTGTITAAAATGAGYCT }\end{array}$ & 129001 & 129042 & HP0118 & $\leftarrow$ & hypothetical protein & not calculated \\
\hline 2 & 61 & $\begin{array}{l}\text { KTCTITGGGTTTGTAAAAATTA } \\
\text { CCTCCTTAATTTGGTITGTITT } \\
\text { GTTAAACTTAAC }\end{array}$ & 425976 & 426036 & HP0413 & $\leftarrow$ & transposase-like protein, PS3IS & not calculated \\
\hline 3 & 14 & GGATGGGTGCTITI & 1475703 & 1475716 & HPrrnB23S & $\leftarrow$ & transposase-like protein, PS3IS & not calculated \\
\hline 4 & 16 & NATCCGCGCTTAAGCG & 1480568 & 1480583 & HP1410 & $\rightarrow$ & hypothetical protein & not calculated \\
\hline Total & 133 & & & & & & & \\
\hline
\end{tabular}


Table 3 SNVs of ATCC26695 sequence data from sample 6

\begin{tabular}{|c|c|c|c|c|c|c|c|}
\hline & $\begin{array}{l}\text { Nucleotide position } \\
\text { (NC_000915) }\end{array}$ & $\begin{array}{l}\text { Reference nucleotide } \\
\text { (NC_000915) }\end{array}$ & $\begin{array}{l}\text { Mutated } \\
\text { nucleotide }\end{array}$ & $\begin{array}{l}\text { Total } \\
\text { reads }\end{array}$ & $\begin{array}{l}\text { Reads of mutated } \\
\text { nucleotide }\end{array}$ & $\begin{array}{l}\text { Frequency of mutated } \\
\text { nucleotide (\%) }\end{array}$ & Annotations \\
\hline 1 & 130106 & T & $C$ & 217 & 117 & 53.9 & $\begin{array}{l}\text { Gene: } \\
\text { HP0119 }\end{array}$ \\
\hline 2 & 130111 & T & G & 186 & 94 & 50.5 & $\begin{array}{l}\text { Gene: } \\
\text { HP0119 }\end{array}$ \\
\hline 3 & 301322 & G & T & 109 & 108 & 99.0 & $\begin{array}{l}\text { Gene: } \\
\text { HP0289 }\end{array}$ \\
\hline 4 & 1370263 & G & $C$ & 101 & 99 & 98.0 & Gene: rpmJ \\
\hline 5 & 1428910 & C & $A$ & 137 & 104 & 75.9 & $\begin{array}{l}\text { Gene: } \\
\text { HP1366 }\end{array}$ \\
\hline 6 & 1502547 & T & $C$ & 345 & 184 & 53.3 & \\
\hline 7 & 1502549 & A & G & 345 & 184 & 53.3 & \\
\hline
\end{tabular}

Total reads and mutated reads indicate the number of reads that passed quality control.

Coding sequence (CDS) variants in efflux pump genes

To identify other possible variants in these clinical isolates, we next examined genetic variants of four gene clusters of TolC homologues, which have been associated with multidrug resistance [23]. To the best of our knowledge, this is the first survey of $H$. pylori MDR efflux transporter variants. During the filtering of uncertain indels and the analysis of $>99.0 \%$ of mapped reads, we created a list of high-quality informative SNVs associated with CDS variants (Table 5).

In 4 of the 7 susceptible strains $(177,189$, F44, and F65), there were no SNVs in the MDR efflux pump genes. The other 3 susceptible strains had SNVs in efflux pump genes. In the CLR-susceptible strain 179, there was a total of 350 SNVs across all four clusters of efflux pump genes. In contrast, all CLR-resistant $H$. pylori strains have SNVs in at

Table 4 Whole-genome sequencing of 19 clinical $H$. pylori isolates

\begin{tabular}{|c|c|c|c|c|c|c|}
\hline Strain & $\begin{array}{l}\text { Total reads } \\
\text { (before trimming) }\end{array}$ & $\begin{array}{l}\text { Total reads } \\
\text { (after trimming) }\end{array}$ & $\begin{array}{l}\text { Total consensus } \\
\text { length (bp) }\end{array}$ & $\begin{array}{l}\text { Total consensus } \\
\text { coverage }(\%)\end{array}$ & $\begin{array}{l}\text { Average coverage } \\
\text { (fold) }\end{array}$ & $\begin{array}{l}\mathrm{NCBI} \text { accession } \\
\text { number }\end{array}$ \\
\hline \multicolumn{7}{|c|}{ CLR-susceptible } \\
\hline 177 & $2,619,844$ & $2,563,352$ & $1,666,155$ & 99.89 & 116.3 & DRS013118 \\
\hline 179 & $2,291,578$ & $2,269,154$ & $1,560,395$ & 93.55 & 131.8 & DRS013119 \\
\hline 189 & $2,010,838$ & $1,967,720$ & $1,664,913$ & 99.82 & 90.9 & DRS013120 \\
\hline F32 & $5,383,222$ & $5,327,650$ & $1,593,944$ & 95.56 & 283.6 & DRS013121 \\
\hline F44 & $3,887,082$ & $3,806,902$ & $1,667,084$ & 99.95 & 157.9 & DRS013122 \\
\hline F65 & $4,248,748$ & $4,157,272$ & $1,667,173$ & 99.95 & 189.6 & DRS013123 \\
\hline F79 & $3,036,516$ & $3,035,946$ & $1,562,308$ & 93.67 & 190.1 & DRS013124 \\
\hline \multicolumn{7}{|c|}{ CLR-resistant } \\
\hline S1 & $2,271,944$ & $2,271,890$ & $1,571,448$ & 94.21 & 149.2 & DRS013125 \\
\hline S2 & $5,962,628$ & $5,962,428$ & $1,587,965$ & 95.20 & 342.2 & DRS013126 \\
\hline S4 & $4,640,490$ & $4,640,330$ & $1,578,524$ & 94.64 & 262.8 & DRS013127 \\
\hline S8 & $10,869,516$ & $10,868,918$ & $1,610,753$ & 96.57 & 686.3 & DRS013128 \\
\hline S13 & $3,873,394$ & $3,873,000$ & $1,563,976$ & 93.77 & 264.2 & DRS013129 \\
\hline S16 & $6,782,392$ & $6,781,628$ & $1,594,857$ & 95.62 & 448.2 & DRS013130 \\
\hline S17 & $5,700,038$ & $5,699,336$ & $1,596,480$ & 95.71 & 327.3 & DRS013131 \\
\hline S22 & $6,597,254$ & $6,596,450$ & $1,598,844$ & 95.86 & 426.8 & DRS013132 \\
\hline S23 & $1,820,652$ & $1,820,436$ & $1,559,100$ & 93.47 & 125.5 & DRS013133 \\
\hline S26 & $6,692,038$ & $6,691,390$ & $1,584,702$ & 95.01 & 442.9 & DRS013134 \\
\hline 174 & $3,004,008$ & $3,003,900$ & $1,580,603$ & 94.76 & 169.7 & DRS013135 \\
\hline 194 & $6,936,714$ & $6,921,552$ & $1,600,238$ & 95.94 & 415.2 & DRS013136 \\
\hline
\end{tabular}

Total reads after trimming are mapped to the reference nucleotide of ATCC26695 and calculated with Genomic Workbench 6.0.1. 


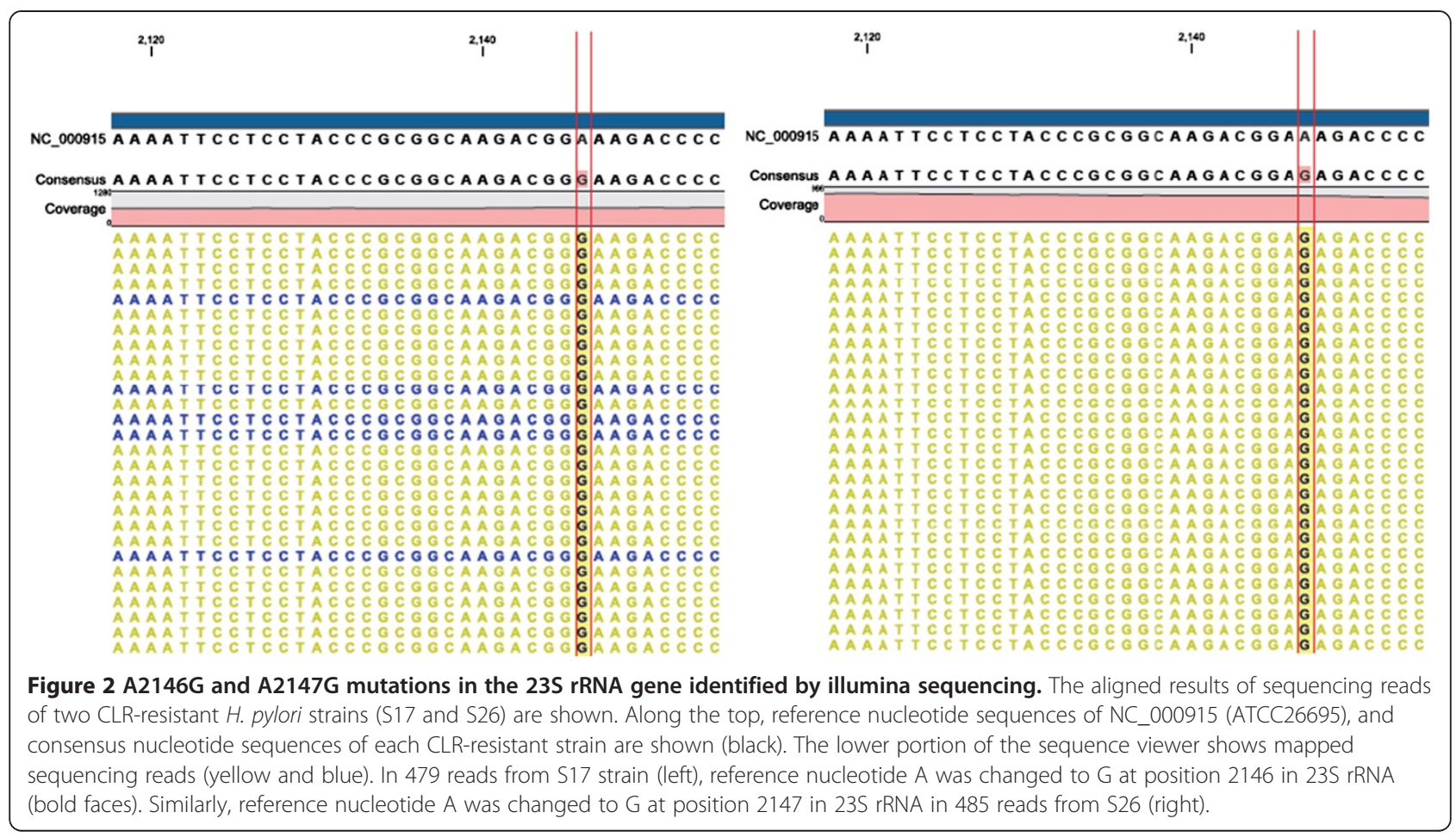

least one of the four gene clusters. Particularly, in the hp0605-hp0607 cluster, SNVs were certainly observed in all 12 resistant strains. There were significant differences in SNVs of the $h p 0605-h p 0607$ cluster between susceptible and resistant strains $(\mathrm{P}<0.05)$, but not in the other clusters.

Correlations between the total number of SNVs in efflux pump clusters and MICs of CLR were examined. In 10 resistant strains out of 12 isolates, MICs of CLR were ranged from 4 to $64 \mu \mathrm{g} / \mathrm{ml}$ and exhibited over $1 \mu \mathrm{g} / \mathrm{ml}$ with the criteria of CLR resistance (Table 5). We found no significant correlation between SNVs and level of CLR resistance.

To examine the common CDS variant in CLR-resistant H. pylori, further detailed analysis of the SNVs in the hp0605-hp0607 cluster was performed (Table 6). In all CLR-resistant strains, there were no common SNVs in each $h p 0605, h p 0606$, and $h p 0607$ cluster. For the maximum number of common SNVs, four SNVs of hp0605 (hefA) were found in 11 out of 12 resistant strains (91.6\%). Of note, one CDS variant of Asn177Thr was in 11 resistant strains. In $h p 0606$ and $h p 0607$, there were no CDS variants specific to the resistant strains, though common SNVs in 11 strains (three SNVs in hp0606 and two SNVs in hp0607) were found. These results indicated that independent SNVs in the efflux pump genes may contribute to CLR susceptibility profiles for each strain.

\section{Discussion}

Here, we have sequenced whole genomes of $H$. pylori isolates by using the massively parallel illumina-based platform. This MiSeq platform can generate the largest amount of paired-end sequence data per sample using a chip-based bridge amplification procedure followed by sequencing by synthesis [24]. To determine the adequate total reads and average coverage per sample, six different sequencing data sets from ATCC26695 have been analyzed. We have observed that the amount of sequencing reads after trimming was directly proportional to the average coverage from 4.2- to 81.7-fold. Genome depth is a major factor that determines the ability to reconstruct genome sequences and detect genetic variants with low error rate [25]. To reach the necessary balance between the number of total reads and average coverage, over one million reads with depth of over 70-fold is sufficient for identification of genetic variants in the bacterial genome of $1.6 \mathrm{Mb}$ [26,27].

However, we could not create a complete gap closure to the reference $H$. pylori genome with sufficient sequencing coverage. Illumina sequencing technique produces large amounts of short overlapping reads from the target genome [28]. The first task of analysis is to assemble these reads to larger regions of the bacterial genome. Since there is an assembly gap, we have reduced the number of false positive candidates and did not try to identify insertions or deletions (indels) of any size in the analysis. Sequencing-based indels detection methods are not well-established even utilizing paired-end data [29], and the reliable detection of indels is complex. In addition, under our approach of reference based assembly, it is difficult to detect true indels due to small assembly gaps even when sequencing the reference genome. Thus, 
Table 5 SNVs of multidrug resistant efflux pump genes and MICs of CLR

\begin{tabular}{|c|c|c|c|c|c|c|c|c|c|c|c|c|c|c|}
\hline Strain & $\begin{array}{l}\text { MICs of CLR } \\
(\mu \mathrm{g} / \mathrm{ml})\end{array}$ & $\begin{array}{l}\text { hp0605 } \\
\text { (hefA) }\end{array}$ & $\begin{array}{l}\text { hp0606 } \\
\text { (hefB ) }\end{array}$ & $\begin{array}{l}\text { hp0607 } \\
\text { (hefC) }\end{array}$ & $\begin{array}{l}\text { hp0969 } \\
\text { (hefF) }\end{array}$ & $\begin{array}{l}\text { hp0970 } \\
\text { (hefE ) }\end{array}$ & $\begin{array}{l}\text { hp097 } 1 \\
\text { (hefD ) }\end{array}$ & $\begin{array}{l}\text { hp1327 } \\
\text { (hefG) }\end{array}$ & $\begin{array}{l}\text { hp1328 } \\
\text { (hefH) }\end{array}$ & $\begin{array}{l}\text { hp1329 } \\
\text { (hefl) }\end{array}$ & hp1487 & hp1488 & hp1489 & Total \\
\hline \multicolumn{15}{|c|}{ CLR-susceptible } \\
\hline 177 & - & 0 & 0 & 0 & 0 & 0 & 0 & 0 & 0 & 0 & 0 & 0 & 0 & 0 \\
\hline 179 & - & 29 & 12 & 70 & 51 & 13 & 3 & 22 & 16 & 47 & 30 & 26 & 31 & 350 \\
\hline 189 & - & 0 & 0 & 0 & 0 & 0 & 0 & 0 & 0 & 0 & 0 & 0 & 0 & 0 \\
\hline F32 & - & 48 & 20 & 76 & 83 & 24 & 27 & 0 & 0 & 0 & 0 & 0 & 0 & 278 \\
\hline F44 & - & 0 & 0 & 0 & 0 & 0 & 0 & 0 & 0 & 0 & 0 & 0 & 0 & 0 \\
\hline F65 & - & 0 & 0 & 0 & 0 & 0 & 0 & 0 & 0 & 0 & 0 & 0 & 0 & 0 \\
\hline F79 & - & 39 & 15 & 49 & 55 & 16 & 30 & 0 & 0 & 0 & 0 & 0 & 0 & 204 \\
\hline \multicolumn{15}{|c|}{ CLR-resistant } \\
\hline S1 & 32 & 32 & 17 & 51 & 55 & 9 & 25 & 23 & 18 & 61 & 23 & 13 & 27 & 354 \\
\hline S2 & 32 & 50 & 18 & 74 & 0 & 0 & 0 & 0 & 0 & 0 & 0 & 0 & 0 & 142 \\
\hline S4 & 32 & 39 & 18 & 67 & 55 & 16 & 26 & 0 & 0 & 0 & 0 & 0 & 0 & 221 \\
\hline S8 & 4 & 44 & 19 & 67 & 0 & 0 & 0 & 0 & 0 & 0 & 0 & 0 & 0 & 130 \\
\hline $\mathrm{S} 13$ & 16 & 52 & 21 & 80 & 76 & 22 & 31 & 0 & 0 & 0 & 0 & 0 & 0 & 282 \\
\hline S16 & 16 & 54 & 23 & 81 & 0 & 0 & 0 & 0 & 0 & 0 & 0 & 0 & 0 & 158 \\
\hline S17 & 32 & 48 & 19 & 87 & 0 & 0 & 0 & 0 & 0 & 0 & 0 & 0 & 0 & 154 \\
\hline S22 & 64 & 46 & 16 & 69 & 0 & 0 & 0 & 0 & 0 & 0 & 0 & 0 & 0 & 131 \\
\hline S23 & 16 & 1 & 16 & 21 & 14 & 2 & 8 & 38 & 10 & 71 & 23 & 19 & 42 & 265 \\
\hline S26 & 32 & 55 & 24 & 83 & 0 & 0 & 0 & 0 & 0 & 0 & 0 & 0 & 0 & 162 \\
\hline 174 & - & 40 & 11 & 55 & 58 & 19 & 34 & 26 & 10 & 52 & 27 & 19 & 47 & 398 \\
\hline 194 & - & 25 & 7 & 34 & 48 & 15 & 18 & 0 & 0 & 0 & 0 & 0 & 0 & 147 \\
\hline \multicolumn{15}{|c|}{ Statistics } \\
\hline$P$ value & & 0.011 & 0.017 & 0.038 & NS & NS & NS & NS & NS & NS & NS & NS & NS & NS \\
\hline $95 \% \mathrm{Cl}$ & & $6.1-41.7$ & $2.5-18.8$ & $2.5-69.9$ & & & & & & & & & & \\
\hline
\end{tabular}

NS; not significant.

we have selected a conservative analysis method focused on SNVs of H. pylori coding sequence.

In the present study, we have applied the AS-PCR method to detect mutations in the 23S rRNA gene of $H$. pylori, in which these mutations were perfectly identical in all sequencing reads at the AS-PCR assayed position. AS-PCR utilizes allele-specific primers such that the second nucleotide from the $3^{\prime}$ end is designed to match the site of the point mutation [30]. This AS-PCR assay is well-established to definitely identify whether a H. pylori strain is sensitive or resistant to CLR [31,32]. Our analysis is a successful comparison between point mutations detected by both AS-PCR and short-read sequencing. Currently, there are few studies in which genetic mutations of antimicrobial resistance identified with sequencing data are consistent with recorded variants $[33,34]$. In addition, next-generation sequencing could clearly distinguish a point mutation in the 23S rRNA gene in the H. pylori genome.

To provide future advances for treatment of H. pylori infections, we need to know whether variants of
MDR efflux pump genes rely on drug resistance as a species-dependent intrinsic factor. The resistancenodulation-cell division (RND) family is grouped into MDR efflux pumps in gram-negative bacteria [35]. AcrB protein, a member of the RND family, forms a homotrimer with an outer membrane protein (TolC) and a periplasmic membrane fusion protein (AcrA) [36,37]. In H. pylori, two TolC homologs, hp0605 and $h p 1489$, have been identified on the basis of structural similarities with outer membrane efflux protein formed RND domains [38]. In addition, three RND efflux systems, each of which consists of an accessory protein of translocase, have been identified as TolC homolog [39]. Now, four gene clusters of efflux pump systems (hp0605-hp0607, hp0971-hp0969, hp1327-hp1329, and hp1489-hp1487) are identified in H. pylori [23] and possibly contribute to resistance to antimicrobials, which resemble the situation in other bacteria [40]. Expression analysis in clinical isolates has shown the importance of efflux pump genes to antimicrobial agents. In response to the exposure of metronidazole, 
Table 6 SNVs of hp0605-hp0607 cluster in 12 CLR-resistant H. pylori

\begin{tabular}{|c|c|c|c|c|c|c|c|c|c|}
\hline \multirow[b]{6}{*}{ Strain } & Locus tag & \multicolumn{8}{|l|}{ HP0605 (NP_207400.1) } \\
\hline & Annotation & \multicolumn{8}{|l|}{ hypothetical protein } \\
\hline & Coding sequence & \multicolumn{2}{|l|}{ C. $530 \mathrm{~A}>\mathrm{C}$} & \multicolumn{2}{|l|}{ c.546A > G } & \multicolumn{2}{|l|}{$\mathrm{c} .804 \mathrm{~T}>\mathrm{C}$} & \multicolumn{2}{|l|}{ c.1365 T > C } \\
\hline & Amino acid & \multicolumn{2}{|l|}{ p.Asn177Thr } & \multicolumn{2}{|l|}{ No } & \multicolumn{2}{|l|}{ No } & \multicolumn{2}{|l|}{ No } \\
\hline & Nucleotide position ${ }^{a}$ & \multicolumn{2}{|l|}{641828} & \multicolumn{2}{|l|}{641844} & \multicolumn{2}{|l|}{642102} & \multicolumn{2}{|l|}{642663} \\
\hline & & $\begin{array}{l}\text { Count reads/ } \\
\text { Total reads }\end{array}$ & Frequency (\%) & $\begin{array}{l}\text { Count reads/ } \\
\text { Total reads }\end{array}$ & Frequency (\%) & $\begin{array}{l}\text { Count reads/ } \\
\text { Total reads }\end{array}$ & Frequency (\%) & $\begin{array}{l}\text { Count reads/ } \\
\text { Total reads }\end{array}$ & Frequency (\%) \\
\hline S1 & & $179 / 180$ & 99.4 & $181 / 181$ & 100 & $140 / 141$ & 99.3 & $107 / 107$ & 100 \\
\hline S2 & & $373 / 373$ & 100 & $392 / 394$ & 99.5 & $319 / 320$ & 99.7 & $295 / 295$ & 100 \\
\hline S4 & & $212 / 213$ & 99.5 & $219 / 221$ & 99.1 & $212 / 214$ & 99.1 & $204 / 205$ & 99.5 \\
\hline S8 & & $507 / 508$ & 99.8 & $530 / 532$ & 99.6 & $478 / 479$ & 99.8 & $503 / 506$ & 99.4 \\
\hline S13 & & $218 / 218$ & 100 & $225 / 225$ & 100 & 169/170 & 99.4 & $179 / 179$ & 100 \\
\hline S16 & & $343 / 343$ & 100 & $371 / 371$ & 100 & $325 / 325$ & 100 & $312 / 313$ & 99.7 \\
\hline S17 & & $266 / 266$ & 100 & $306 / 306$ & 100 & 185/186 & 99.5 & $246 / 246$ & 100 \\
\hline S22 & & $328 / 328$ & 100 & $348 / 348$ & 100 & 299/299 & 100 & $287 / 289$ & 99.3 \\
\hline S23 & & - & & - & & - & & - & \\
\hline S26 & & $341 / 341$ & 100 & $368 / 368$ & 100 & $321 / 321$ & 100 & $310 / 311$ & 99.7 \\
\hline 174 & & $123 / 123$ & 100 & $124 / 124$ & 100 & $114 / 114$ & 100 & $151 / 151$ & 100 \\
\hline 194 & & $305 / 306$ & 99.7 & $324 / 324$ & 100 & $341 / 343$ & 99.4 & $231 / 232$ & 99.6 \\
\hline & Locus tag & HP0606 (NP_207401.1) & & & & & & & \\
\hline & Annotation & membrane fusion protein MtrC & & & & & & & \\
\hline & Coding sequence & C.99 T > C & & c. $105 \mathrm{~A}>\mathrm{G}$ & & c. $192 A>G$ & & & \\
\hline & Amino acid & No & & No & & No & & & \\
\hline & Nucleotide position & 642841 & & 642847 & & 642934 & & & \\
\hline Strain & & $\begin{array}{l}\text { Count reads/ } \\
\text { Total reads }\end{array}$ & Frequency (\%) & $\begin{array}{l}\text { Count reads/ } \\
\text { Total reads }\end{array}$ & Frequency (\%) & $\begin{array}{l}\text { Count reads/ } \\
\text { Total reads }\end{array}$ & Frequency (\%) & & \\
\hline S1 & & $140 / 140$ & 100 & $129 / 129$ & 100 & $139 / 140$ & 99.3 & & \\
\hline S2 & & $340 / 340$ & 100 & $324 / 324$ & 100 & $383 / 385$ & 99.5 & & \\
\hline S4 & & 194/194 & 100 & 192/193 & 99.5 & $221 / 223$ & 99.1 & & \\
\hline S8 & & $496 / 498$ & 99.6 & $472 / 473$ & 99.8 & $505 / 506$ & 99.8 & & \\
\hline S13 & & $203 / 203$ & 100 & 194/194 & 100 & - & & & \\
\hline S16 & & $329 / 331$ & 99.4 & $316 / 317$ & 99.7 & $335 / 335$ & 100 & & \\
\hline S17 & & $233 / 233$ & 100 & $230 / 230$ & 100 & $272 / 273$ & 99.6 & & \\
\hline S22 & & $295 / 295$ & 100 & $285 / 285$ & 100 & $320 / 322$ & 99.4 & & \\
\hline
\end{tabular}


Table 6 SNVs of hp0605-hp0607 cluster in 12 CLR-resistant H. pylori (Continued)

\begin{tabular}{|c|c|c|c|c|c|c|c|}
\hline S23 & & 109/109 & 100 & $105 / 105$ & 100 & $110 / 110$ & 100 \\
\hline S26 & & $323 / 325$ & 99.4 & $311 / 312$ & 99.7 & $331 / 331$ & 100 \\
\hline 174 & & 137/138 & 99.3 & $126 / 127$ & 99.2 & 137/138 & 99.3 \\
\hline 194 & & - & & - & & 299/301 & 99.3 \\
\hline & Locus tag & HP0607 (NP_207402.1) & & & & & \\
\hline & Annotation & acriflavine resistance protein AcrB & & & & & \\
\hline & Coding sequence & C. $1341 \mathrm{~T}>\mathrm{C}$ & & c.39A $>G$ & & & \\
\hline & Amino acid & No & & No & & & \\
\hline & Nucleotide position & 644800 & & 643498 & & & \\
\hline Strain & & Count reads/Total reads & Frequency (\%) & Count reads/Total reads & Frequency (\%) & & \\
\hline S1 & & $159 / 160$ & 99.4 & $123 / 124$ & 99.2 & & \\
\hline S2 & & $345 / 347$ & 99.4 & $280 / 280$ & 100 & & \\
\hline S4 & & $284 / 286$ & 99.3 & $182 / 182$ & 100 & & \\
\hline S8 & & $585 / 586$ & 99.8 & $431 / 432$ & 99.8 & & \\
\hline S13 & & $227 / 228$ & 99.6 & $164 / 164$ & 100 & & \\
\hline S16 & & $425 / 425$ & 100 & 295/295 & 100 & & \\
\hline S17 & & $317 / 317$ & 100 & 198/198 & 100 & & \\
\hline S22 & & $346 / 347$ & 99.7 & $273 / 275$ & 99.3 & & \\
\hline S23 & & $104 / 104$ & 100 & 106/106 & 100 & & \\
\hline S26 & & $419 / 419$ & 100 & 295/295 & 100 & & \\
\hline 174 & & $113 / 113$ & 100 & - & & & \\
\hline 194 & & - & & $342 / 344$ & 99.4 & & \\
\hline
\end{tabular}

${ }^{a}$ Nucelotide position was based on NC_000915. 
expression of TolC efflux pump gene (hp0605) has been shown to increase, indicating the relation with drug resistance in $H$. pylori [41]. More recently, exposure of CLR in vitro has induced natural transformation to the genome of strain 26695, which resulted in the point mutation of acrB ( $\mathrm{pp0607)}$ with whole-genome sequencing [42]. To extend these previous findings, this study highlights the importance of MDR efflux pumps of $H$. pylori derived from active infections.

This study has indicated a link between specific variants of efflux pump genes and the CLR-resistant phenotype. The exact mechanism underlying these genetic mutations is unclear. Possible mechanisms of intrinsic drug resistance involve decreased drug uptake or increased drug efflux [39]. Knockout mutants for each of hp0605 and hp0971 have displayed drug susceptibility profiles to metronidazole, novobiocin, and sodium deoxycholate [23]. Indeed, we have found that specific mutations in $h p 0605$, hp0971, and the other variants in efflux pump systems are significant in CLR-resistant strains. Whole-genome sequencing data include vast amounts of additional information that are currently unavailable from small scale analysis. Variants in efflux pump genes have a possible effect on the association with drug susceptibility in clinical isolates.

\section{Conclusions}

We have provided successful validation of genotypic prediction for antimicrobial resistance with the PCR-based approach and highlighted the potential of whole-genome sequencing in the analysis of $H$. pylori isolates. These sequencing data have emphasized the significant function of the variants of efflux pump genes in CLR-resistant strains. Since we have been conservative in our analysis to focus on only well-validated SNVs in limited parts of the bacterial genome, further analyses (e.g., indels, short repeats, rearrangements, and phylogenetics) with whole genome methods, such as gap closure, could provide more detailed discrimination of the drug resistant $H$. pylori isolates.

\section{Methods}

\section{H.pylori samples}

Nineteen H. pylori clinical isolates were separated from gastric epithelium biopsy tissues during upper gastroduodenal endoscopy at Okinawa Prefectural Chubu Hospita, and Kobe University Hospital. All patients gave written informed consent for use of their samples for the present study, and this study was performed according to the principles of the Declaration of Helsinki. The major reference strain, ATCC26695 (NC_000915), was also used.

\section{H.pylori culture}

Gastric biopsy specimens were transferred onto a Trypticase soy agar II (TSA-II)-5\% sheep blood plate and cultured under microaerobic conditions $\left(\mathrm{O}_{2}, 5 \% ; \mathrm{CO}_{2}, 15 \% ; \mathrm{N}_{2}\right.$,
$80 \%)$ at $37^{\circ} \mathrm{C}$ for 5 days. One colony was picked from each primary culture plate, and seeded onto a fresh TSA-II plate. A few colonies were picked from each plate and transferred into $2 \mathrm{ml}$ of brucella broth liquid culture medium containing $10 \%$ fetal calf serum and cultured for 3 days. A part of the liquid culture was stored at $-80^{\circ} \mathrm{C}$ in 0.01 M PBS containing 20\% glycerol. $H$. pylori DNA was extracted from the pellet of the liquid culture by the protease-phenol-chloroform method, suspended in $300 \mu \mathrm{l}$ of TE buffer, and stored at $4^{\circ} \mathrm{C}$ until AS-PCR and sequencing.

\section{Antibiotic susceptibility testing}

To determine the MICs of CLR, CLSI agar dilution method was used [43]. Briefly, two-fold dilutions of CLR were prepared in Muller-Hinton agar (Becton Dickinson, MD) with $5 \%$ sheep blood. Following the preparation of H. pylori suspension in physiological saline adjusted to a 2.0 McFarland standard, a 1 to $3 \mu \mathrm{l}$ inoculum was spotted on agar plate. Following incubation at $37^{\circ} \mathrm{C}$ for $72 \mathrm{~h}$ under microaerophilic atomosphere, the MICs of CLR were established as the drug concentration showing no growth.

Allele-specific PCR (AS-PCR) for 235 rRNA gene mutations Analysis of alleles with point mutations in 23S rRNA (A2146G and A2147G) was performed according to previous reports [31,32]. The allele-specific primer sequences are listed in Additional file 4: Table S1. The PCR mixture was contained $100 \mathrm{ng}$ of DNA with $10 \times$ buffer $2.5 \mu \mathrm{l}$, $2 \mathrm{mM}$ dNTP $2.5 \mu \mathrm{l}, 25 \mathrm{mM} \mathrm{MgCl} 21 \mu \mathrm{l}, 10$ pmol primer mixtures, and 0.5 U KOD-plus DNA polymerase (TOYOBO, Osaka, Japan). Then, mixture was up to $25 \mu \mathrm{l}$ with distilled water. The PCR condition was as follows; $94^{\circ} \mathrm{C}$ for $2 \mathrm{~min}$ and 35 cycles of $94^{\circ} \mathrm{C}$ for $15 \mathrm{sec}, 60^{\circ} \mathrm{C}$ for $30 \mathrm{sec}$, and $68^{\circ} \mathrm{C}$ for $30 \mathrm{sec}$, followed by $68^{\circ} \mathrm{C}$ for $2 \mathrm{~min}$. PCR products were determined by $4 \%$ agarose gel electrophoresis. The gels were stained with ethidium bromide to determine the product size. ATCC26695 and 599 were used as control strains for the AS-PCR.

\section{Preparation of genomic DNA and whole-genome sequencing}

Total DNA of $H$. pylori isolated from patients and the reference ATCC26695 were sequenced. Bacterial DNA was extracted with DNeasy blood and tissue kit according to manufacturer's guideline (Qiagen, Hilden, Germany). DNA concentration of each sample was measured with Qubit dsDNA HS assay kit (Q32851; Invitrogen, Carlsbad, CA). An $<500$-bp DNA library of $H$. pylori strains (50 ng or $1 \mathrm{ng}$ ) was prepared by using Nextera DNA Sample Prep Kit (illumina, San Diego, CA) or Nextera XT DNA Sample Prep Kit (illumina). According to the manufacturer's instructions (version May 2012), DNA was uniformly sheared into 500-bp portions with these kits, and DNA libraries 
were pooled with $1 \% 8$ pM PhiX control Spike in, and then run on MiSeq sequencer (illumina) with Reagent kit (300 cycle, paired-end). Fluorescent images were analyzed using the MiSeq Control Software, and FASTQ-formatted sequence data was created using MiSeq Reporter Analysis.

\section{Sequence reads mapping and SNVs detection}

For used sequence data, read quality was selected at Q30 over $80 \%$ followed by the suggestion of illumina. After quality check and data trimming, Genomics Workbench 6.0.1 (CLC bio, Aarhus, Denmark) was used to assemble the sequence. The read mapping module was termed as CLC Assembly Cell 4.0, which was based on an uncompressed Suffix-Array representing the entire reference genome in a single data structure (White paper on CLC read mapper; October 10, 2012). Sequence reads were mapped against the reference genome of ATCC26695 (NC_000915), and single nucleotide variants (SNVs) were identified with probabilistic variant detection modules with default parameters and minor modifications in the mapping algorithm. Variant detection was set to 1 in Genomics Workbench 6.0.1. To exclude false-positive variants resulting from sequencing errors, we selected variants presented in $>99.0 \%$ of mapped reads with minimum coverage of 100 . Insertions and deletions were also discarded. During this process, a set of high confidence variants was generated.

\section{Variants of MDR efflux pumps in clinical $H$. pylori strains}

Generally, in gram-negative bacteria, the resistancenodulation-cell division (RND) family is representative of MDR efflux pumps, which includes the AcrAB-TolC system in Escherichia coli [35]. In the H. pylori 26695 genome, four gene clusters of TolC homologues, hp0605- $h p 0607$ (hefABC), hp0971-hp0969 (hefDEF), hp1327-hp1329 (hefGHI), and $h p 1489-h p 1487$, have been indicated as MDR efflux pumps based on sequence similarity to other bacterial species [23] and their gene expression patterns [16].

\section{Statistical analysis}

The associations between the genetic variants of efflux pump genes and the phenotype of CLR resistance were analyzed with Student's t-test. Correlation coefficient between the genetic variants and MICs of CLR was also calculated, and the 95\% confidence interval (CI) was used to estimate the risk. A P value of $<0.05$ was accepted as statistically significant. The SPSS statistical software package version 20.0 (SPSS, Inc., Chicago, IL) was used for all statistical analyses.

\section{Nucleotide sequence accession number}

All sequence reads of 19 clinical isolates and ATCC26695 have been deposited in the DNA Data Bank of Japan Sequence Read Archive (http://www.ddbj.nig.ac.jp/indexe.html) under accession number DRA001250.

\section{Additional files}

Additional file 1: Figure S1. Sequencing quality control of ATCC26695 sequence data from sample 6 . ATCC26695 sequence data from sample 6 with $2,148,576$ reads was subjected to the quality control analysis. The length distribution of sequencing reads (A), ambiguous base-content (B), and quality distribution at each base position (C), are shown.

Additional file 2: Figure S2. Flow chart of sequencing reads analysis. Additional file 3: Figure S3. Sequence of 235 rRNA gene at 2146 and 2147 position in CLR-susceptible F79 strain.

Additional file 4: Table S1. Allele specific-PCR primer sequences.

\section{Competing interests}

The authors declared that they have no competing interests.

\section{Authors' contributions}

$\Pi$, Al, and $R O$ conceived and designed the research. Al, RO, LY, and $K Y$ collected samples and performed experiments. TT, Al, and RO analyzed the data and prepared figures, interpreted results of experiments, and drafted manuscript. TT and Al edited manuscript. YM, SN, MY, and TA supervised this study. All authors read and approved the final manuscript.

\section{Acknowledgements}

This study was supported by a Grant-in-Aid (no. 26460212) to $\Pi$ from the Ministry of Education, Culture, Sports, Science and Technology of Japan, JSPS Grant-in-Aid for Scientific Research on Innovative Areas (no. 22114006) to TA, JSPS Grants-in-Aid for Scientific Research (no. 25305027) to TA, and JSPS Asian CORE Program to TA. TT and YM were financially supported by the Ministry of Health, Labour, and Welfare of Japan (H25-general-008). This study was also supported by Toyobo Co. Ltd. (Osaka, Japan).

\section{Author details}

${ }^{1}$ Department of Internal Medicine, Division of Gastroenterology, Kobe University Graduate School of Medicine, 7-5-1 Kusunoki-cho, Chuo-ku, Kobe 650-0017, Japan. ²Department of Medical Pharmaceutics, Kobe

Pharmaceutical University, 4-19-1 Motoyama-kita, Higashinada-ku, Kobe 658-8558, Japan. ${ }^{3}$ Division of Internal Medicine, Okinawa Prefectural Chubu Hospital, 281 Miyazato, Uruma-city, Okinawa 904-2293, Japan. ${ }^{4}$ Department of Hepatology, Osaka City University Graduate School of Medicine, 1-4-3 Asahimachi, Abeno-ku, Osaka 545-8585, Japan.

Received: 15 May 2014 Accepted: 18 June 2014

Published: 26 June 2014

\section{References}

1. Gisbert JP, Calvet X: Review article: the effectiveness of standard triple therapy for Helicobacter pylori has not changed over the last decade, but it is not good enough. Aliment Pharmacol Ther 2011, 34:1255-1268.

2. McColl KE: Clinical practice. Helicobacter pylori infection. N Engl J Med 2010, 362:1597-1604.

3. Malfertheiner P, Megraud F, O'Morain C, Bazzoli F, El-Omar E, Graham D, Hunt R, Rokkas T, Vakil N, Kuipers EJ: Current concepts in the management of Helicobacter pylori infection: the Maastricht III Consensus Report. Gut 2007, 56:772-781.

4. Gisbert JP, Calvet X, Gomollon F, Mones J: [Eradication treatment of Helicobacter pylori. Recommendations of the II Spanish Consensus Conference]. Med Clin (Barc) 2005, 125:301-316.

5. Caselli M, Zullo A, Maconi G, Parente F, Alvisi V, Casetti T, Sorrentino D, Gasbarrini G: "Cervia II Working Group Report 2006": guidelines on diagnosis and treatment of Helicobacter pylori infection in Italy. Dig Liver Dis 2007, 39:782-789.

6. Chey WD, Wong BC: American College of Gastroenterology guideline on the management of Helicobacter pylori infection. Am J Gastroenterol 2007, 102:1808-1825.

7. Jenks PJ: Causes of failure of eradication of Helicobacter pylori. BMJ 2002 325:3-4.

8. Vakil N, Megraud F: Eradication therapy for Helicobacter pylori. Gastroenterology 2007, 133:985-1001. 
9. Graham DY, Lu H, Yamaoka Y: A report card to grade Helicobacter pylori therapy. Helicobacter 2007, 12:275-278.

10. Versalovic J, Shortridge D, Kibler K, Griffy MV, Beyer J, Flamm RK, Tanaka SK, Graham DY, Go MF: Mutations in 23S rRNA are associated with clarithromycin resistance in Helicobacter pylori. Antimicrob Agents Chemother 1996, 40:477-480.

11. Taylor DE, Ge Z, Purych D, Lo T, Hiratsuka K: Cloning and sequence analysis of two copies of a $23 \mathrm{~S}$ rRNA gene from Helicobacter pylori and association of clarithromycin resistance with $23 \mathrm{~S}$ rRNA mutations. Antimicrob Agents Chemother 1997, 41:2621-2628.

12. Rimbara E, Noguchi $N$, Kawai T, Sasatsu M: Novel mutation in $23 S$ rRNA that confers low-level resistance to clarithromycin in Helicobacter pylori. Antimicrob Agents Chemother 2008, 52:3465-3466.

13. Kim JM, Kim JS, Kim N, Kim YJ, Kim IY, Chee YJ, Lee CH, Jung HC: Gene mutations of $23 \mathrm{~S}$ rRNA associated with clarithromycin resistance in Helicobacter pylori strains isolated from Korean patients. J Microbiol Biotechnol 2008, 18:1584-1589.

14. Nash KA, Brown-Elliott BA, Wallace RJ Jr: A novel gene, erm(41), confers inducible macrolide resistance to clinical isolates of Mycobacterium abscessus but is absent from Mycobacterium chelonae. Antimicrob Agents Chemother 2009, 53:1367-1376.

15. Roberts M: Update on macrolide-lincosamide-streptogramin, ketolide, and oxazolidinone resistance genes. FEMS Microbiol Lett 2008, 282:147-159.

16. Hirata K, Suzuki H, Nishizawa T, Tsugawa H, Muraoka H, Saito Y, Matsuzaki J, Hibi T: Contribution of efflux pumps to clarithromycin resistance in Helicobacter pylori. J Gastroenterol Hepatol 2010, 25(Suppl 1):S75-S79.

17. De Francesco V, Margiotta M, Zullo A, Hassan C, Valle ND, Burattini O, D'Angelo R, Stoppino G, Cea U, Giorgio F, Monno R, Morini S, Panella C, lerardi E: Claritromycin resistance and Helicobacter pylori genotypes in Italy. J Microbiol 2006, 44:660-664.

18. Tanuma M, Rimbara E, Noguchi N, Boonyaritichaikij S, Kuwabara K Fukunaga Y, Sasatsu M: Analysis of clarithromycin resistance and CagA status in Helicobacter pylori by use of feces from children in Thailand. $J$ Clin Microbiol 2009, 47:4144-4145.

19. MacLean D, Jones JD, Studholme DJ: Application of 'next-generation' sequencing technologies to microbial genetics. Nat Rev Microbiol 2009, 7:287-296.

20. Didelot $X$, Bowden R, Wilson DJ, Peto TE, Crook DW: Transforming clinical microbiology with bacterial genome sequencing. Nat Rev Genet 2012, 13:601-612.

21. Loman NJ, Constantinidou C, Chan JZ, Halachev M, Sergeant M, Penn CW, Robinson ER, Pallen MJ: High-throughput bacterial genome sequencing: an embarrassment of choice, a world of opportunity. Nat Rev Microbiol 2012, 10:599-606.

22. Koser CU, Ellington MJ, Cartwright EJ, Gillespie SH, Brown NM, Farrington M, Holden MT, Dougan G, Bentley SD, Parkhill J, Peacock SJ: Routine use of microbial whole genome sequencing in diagnostic and public health microbiology. PLoS Pathog 2012, 8:e1002824.

23. van Amsterdam $K$, Bart A, van der Ende A: A Helicobacter pylori TolC efflux pump confers resistance to metronidazole. Antimicrob Agents Chemother 2005, 49:1477-1482.

24. Bentley DR, Balasubramanian S, Swerdlow HP, Smith GP, Milton J, Brown CG, Hall KP, Evers DJ, Barnes CL, Bignell HR, Boutell JM, Bryant J, Carter RJ, Keira Cheetham R, Cox AJ, Ellis DJ, Flatbush MR, Gormley NA, Humphray SJ, Inving LJ Karbelashvili MS, Kirk SM, Li H, Liu X, Maisinger KS, Murray L, Obradovic B, Ost T, Parkinson ML, Pratt MR, et al: Accurate whole human genome sequencing using reversible terminator chemistry. Nature 2008, 456:53-59.

25. Clark MJ, Chen R, Lam HY, Karczewski KJ, Chen R, Euskirchen G, Butte AJ, Snyder M: Performance comparison of exome DNA sequencing technologies. Nat Biotechnol 2011, 29:908-914.

26. Howden BP, McEvoy CR, Allen DL, Chua K, Gao W, Harrison PF, Bell J, Coombs G, Bennett-Wood V, Porter JL, Robins-Browne R, Davies JK, Seemann T, Stinear TP: Evolution of multidrug resistance during Staphylococcus aureus infection involves mutation of the essential two component regulator WalKR. PLoS Pathog 2011, 7:e1002359.

27. Perkins TT, Tay CY, Thirriot F, Marshall B: Choosing a benchtop sequencing machine to characterise Helicobacter pylori genomes. PLoS One 2013, 8:e67539.

28. Quail MA, Smith M, Coupland P, Otto TD, Harris SR, Connor TR, Bertoni A, Swerdlow HP, Gu Y: A tale of three next generation sequencing platforms: comparison of lon Torrent, Pacific Biosciences and Illumina MiSeq sequencers. BMC Genomics 2012, 13:341.

29. Emde AK, Schulz MH, Weese D, Sun R, Vingron M, Kalscheuer VM, Haas SA, Reinert K: Detecting genomic indel variants with exact breakpoints in single- and paired-end sequencing data using SplazerS. Bioinformatics 2012, 28:619-627.

30. Nishizawa T, Suzuki H, Umezawa A, Muraoka H, Iwasaki E, Masaoka T, Kobayashi I, Hibi T: Rapid detection of point mutations conferring resistance to fluoroquinolone in gyrA of Helicobacter pylori by allele-specific PCR. J Clin Microbiol 2007, 45:303-305.

31. Nakamura A, Furuta T, Shirai N, Sugimoto M, Kajimura M, Soya Y, Hishida A: Determination of mutations of the $23 \mathrm{~S}$ rRNA gene of Helicobacter pylori by allele specific primer-polymerase chain reaction method. J Gastroenterol Hepatol 2007, 22:1057-1063.

32. Furuta T, Soya Y, Sugimoto M, Shirai N, Nakamura A, Kodaira C, Nishino M, Okuda M, Okimoto T, Murakami K, Fujioka T, Hishida A: Modified allele-specific primer-polymerase chain reaction method for analysis of susceptibility of Helicobacter pylori strains to clarithromycin. J Gastroenterol Hepatol 2007, 22:1810-1815.

33. McAdam PR, Holmes A, Templeton KE, Fitzgerald JR: Adaptive evolution of Staphylococcus aureus during chronic endobronchial infection of a cystic fibrosis patient. PLoS One 2011, 6:e24301.

34. Mutreja A, Kim DW, Thomson NR, Connor TR, Lee JH, Kariuki S, Croucher NJ, Choi SY, Harris SR, Lebens M, Niyogi SK, Kim EJ, Ramamurthy T, Chun J, Wood JL, Clemens JD, Czerkinsky C, Nair GB, Holmgren J, Parkhill J, Dougan G: Evidence for several waves of global transmission in the seventh cholera pandemic. Nature 2011, 477:462-465.

35. Okusu $H, M a D$, Nikaido $H$ : AcrAB efflux pump plays a major role in the antibiotic resistance phenotype of Escherichia coli multiple-antibiotic-resistance (Mar) mutants. J Bacteriol 1996, 178:306-308.

36. Murakami S, Nakashima R, Yamashita E, Yamaguchi A: Crystal structure of bacterial multidrug efflux transporter AcrB. Nature 2002, 419:587-593.

37. Nakashima R, Sakurai K, Yamasaki S, Nishino K, Yamaguchi A: Structures of the multidrug exporter AcrB reveal a proximal multisite drug-binding pocket. Nature 2011, 480:565-569.

38. Johnson JM, Church GM: Alignment and structure prediction of divergent protein families: periplasmic and outer membrane proteins of bacterial efflux pumps. J Mol Biol 1999, 287:695-715.

39. Bina JE, Alm RA, Uria-Nickelsen M, Thomas SR, Trust TJ, Hancock RE: Helicobacter pylori uptake and efflux: basis for intrinsic susceptibility to antibiotics in vitro. Antimicrob Agents Chemother 2000, 44:248-254.

40. Alvarez-Ortega C, Olivares J, Martinez JL: RND multidrug efflux pumps: what are they good for? Front Microbiol 2013, 4:7.

41. Tsugawa H, Suzuki H, Muraoka H, Ikeda F, Hirata K, Matsuzaki J, Saito Y, Hibi T: Enhanced bacterial efflux system is the first step to the development of metronidazole resistance in Helicobacter pylori. Biochem Biophys Res Commun 2011, 404:656-660.

42. Binh TT, Shiota S, Suzuki R, Matsuda M, Trang TT, Kwon DH, Iwatani S, Yamaoka Y: Discovery of novel mutations for clarithromycin resistance in Helicobacter pylori by using next-generation sequencing. J Antimicrob Chemother 2014. [Epub ahead of print].

43. Clinical and Laboratory Standards Institute: Performance Standards for Antimicrobial Susceptibility Testing: Seventeenth Informational Supplement M100-S17. In CLSI. PA, USA: Wayne; 2007.

doi:10.1186/1757-4749-6-27

Cite this article as: Iwamoto et al: Whole-genome sequencing of clarithromycin resistant Helicobacter pylori characterizes unidentified variants of multidrug resistant efflux pump genes. Gut Pathogens 2014 6:27. 